\title{
Automatic Generation of Model Checking Scripts based on Environment Modeling
}

\author{
Kenro Yatake and Toshiaki Aoki \\ Japan Advanced Institute of Science and Technology, \\ 1-1 Asahidai Nomi Ishikawa 923-1292, Japan \\ $\{k$-yatake, toshiaki\}@jaist.ac.jp
}

\begin{abstract}
When applying model checking to the design models of the embedded systems, it is necessary to model not only the behavior of the target system but also that of the environment interacting with the system. In this paper, we present a method to model the environment and to automatically generate all possible environments from the model. In our method, we can flexibly model the structural variation of the environment and the sequences of the function calls using a class model and statechart models. We also present a tool to generate Promela scripts of SPIN from the environment model. As a practical experiment, we applied our tool to the verification of an OSEK/VDX RTOS design model.
\end{abstract}

\section{Introduction}

Recently, model checking is drawing attention as a technique to improve the reliability of software systems [18]. Especially, they are widely applied to a verification of embedded systems. The major characteristics of embedded systems is reactiveness. i.e., they operate by the stimulus from the environment. For example, Real-Time Operating Systems (RTOS), which are embedded in most of the complex embedded systems, operate by the service calls from the tasks running on them. In order to apply model checking to such systems, it is necessary to model not only the behavior of the target system but also that of the environment.

The most typical approach to model an environment is to construct a process which calls all the functions provided by the system non-deterministically. Although it realizes an exhaustive check for all the possible execution sequences, description of properties tends to become complicated because it needs extra assumptions to filter out uninterested sequences from all the sequences. Furthermore, it is prone to suffer state explosion because all the sequences are checked at a time. Another approach is to call specific sequences of functions depending on the properties to check. For example, we limit the range of the function calls to the normal execution sequences and check that certain properties hold in that range. The advantage of this approach is that the property description becomes simple and precise because the assumptions of the properties are implied by the sequences themselves. This enables us to reflect our intention of verification more 
clearly on the model. Furthermore, as the range is limited, we are more likely to be able to avoid state explosion.

We consider the latter approach is more realistic because state explosion is a critical problem in model checking. However, we must further consider the structural variation of the environment. For example, the environment of an RTOS consists of a multiple number of tasks and resources. There are also various patterns in their priority values and function call relationships. Although we need to check the system for all the environment variations, the number of the variations is so large that we cannot construct them by hand.

To cope with this problem, we propose an environment modeling method where we model the environment variations in a model called environment model and automatically generate all possible environments from the model. To allow the use in practice, we defined the environment model based on UML [10]. In a class model, we can model the structural variation of the environment. In statechart models, we can define the sequences of the function calls. Our method is implemented as a tool called environment generator which inputs an environment model and outputs environments as Promela scripts of SPIN. As a practical experiment, we applied the tool to the verification of an RTOS design model which is based on OSEK/VDX RTOS specification [11]. In this paper, we explain the details of our method and the verification experiment of the RTOS model.

This paper is organized as follows. In section 2, we explain the approach of our method. In section 3, we explain the environment model. In section 4, we explain the environment generation and the tool. In section 5, we show the verification experiment. In section 6 , we discuss the coverage and parallelization of our method. In section 7 , we give a conclusion and future work.

\section{Approach}

Let us explain how reactive systems are verified using an environment. Fig.1 shows an RTOS and its environment. The RTOS implements data structures such as a task control blocks and a ready queue and provides functions such as ActivateTask() and TerminateTask(). If these functions are called, the RTOS schedules the tasks based on their priorities. To verify this behavior, we prepare an environment, for example, consisting of two tasks T1 and T2 (T2's priority is higher than T1's). This environment describes a sequence of function calls and state transitions of the tasks expected by the calls. For example, if the function ActivateTask () is called to T2, T2 is expected to become running. Then, if the same function is called to T1, T1 is expected to become ready. To verify that the RTOS satisfies this expectation, we apply model checking to the RTOS in combination with the environment. Specifically, in each state of the environment, we check the consistency between the environment state and the internal values of the RTOS. For example, if $\mathrm{T} 1$ and $\mathrm{T} 2$ are ready and running, the ready queue in the RTOS must contain the identifier of T1, and the variable turn 

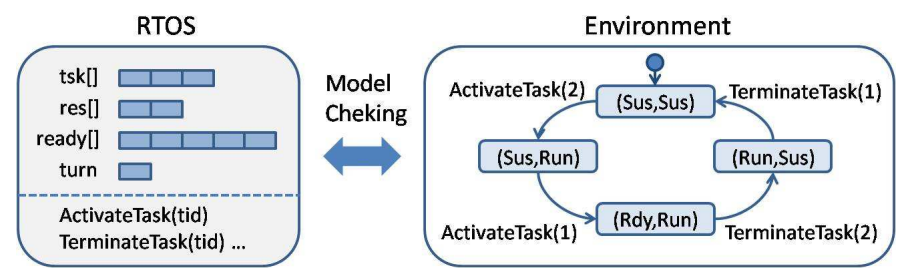

Fig. 1. Model checking with an environment

(representing the running task) must contain the identifier of T2. By checking this consistency, we verify the behavior of the RTOS.

The problem of this approach is that this environment is only one of the cases of the large number of environment variations. We need to verify the RTOS for all the variations with respect to the structures such as the number of tasks and resources, the patterns of the priority values and function call relationships. But it is unrealistic to construct all of them by hand. One could think of constructing a general environment with $m$ tasks and $n$ resources, but this is prone to suffer state explosion.

To cope with this problem, we introduce a model to describe the environment variations and automatically generate all the environments from the model. Fig. 2 summarizes this idea. To verify the target system (RTOS design model), we first construct an environment model. This model is based on UML in which all possible environment structures are defined using parameters with ranges. Then, we generate all the environment instances from the model using the environment generator. All the generated instances, which are structurally different from each other, cover all the variations of the environment. The environment generator inputs an environment model as a text file and outputs the environments as Promela scripts. Finally, we combine the target system with each environment instance and conduct model checking using SPIN.

Our approach has the following advantages:

1. Easy syntax: The syntax of the environment model is based on UML which is familiar to most of the software engineers. This lowers the hurdle for introducing our method to the software development in practice.

2. Alleviation of state explosion problem: It alleviates the state explosion problem by structurally dividing the whole environment into individual environment. As each environment can be checked in a relatively small state space, we are likely to be able to check the whole environment without causing state explosion.

3. Structural difference analysis: When a bug is detected in model checking, our method allows us to structurally analyze its source, i.e., we can identify the structural boundary of the source of a bug by comparing the check results. For example, when the two results "The case of 2 tasks and no resources is correct" and "The case of 2 tasks and 1 resource is not correct" 


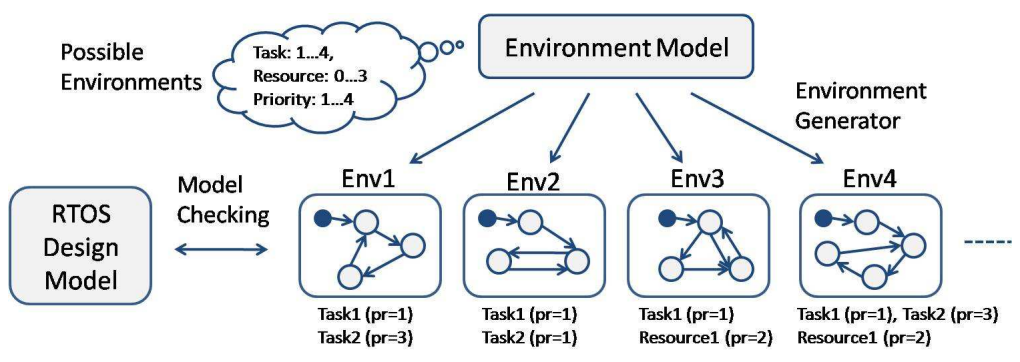

Fig. 2. Environment modeling method

are obtained, we can presume that the resource handling function contains a bug.

4. Generality: It can be generally applied to the verification of reactive systems. Our method is especially effective for the systems whose environment has a lot of structural variations. Examples of such systems are operating systems and middleware systems.

\section{Environment models}

In this section, we explain environment models with an example of RTOS. We also present formal definitions in A.1.

\subsection{Class model}

Fig. 3 shows the class model of the environment for an RTOS. The class model consists of a class representing a target system and classes representing its environment. In the figure, the class RTOS is the target system and the two classes Task and Resource are the environment classes.

The target class defines two kinds of functions as the interface with the environment. The functions labeled with fun are trigger functions. They trigger the state transition of the target system. For example, ActivateTask (tid,dtid) is the function to activate the task of ID dtid (tid is the ID of the caller task). The argument of a function is defined with a range like tid:1..M. representing the variation of the arguments. The functions labeled with ref are reference functions. They refer the internal values of the target system. They are used to define assertions (explained later in this section).

The environment classes are defined with attributes, associations, and variables. They are labelled with attr, assoc, and var, respectively. An attribute is defined with a range like pr:1..P representing the variation of the attribute values. ( $\mathrm{pr}$ is a priority of a task.) An association is also defined with a range like res:0..N. representing the variation of the multiplicity, i.e., the number of objects linked with an object. The associations from the target class to an 


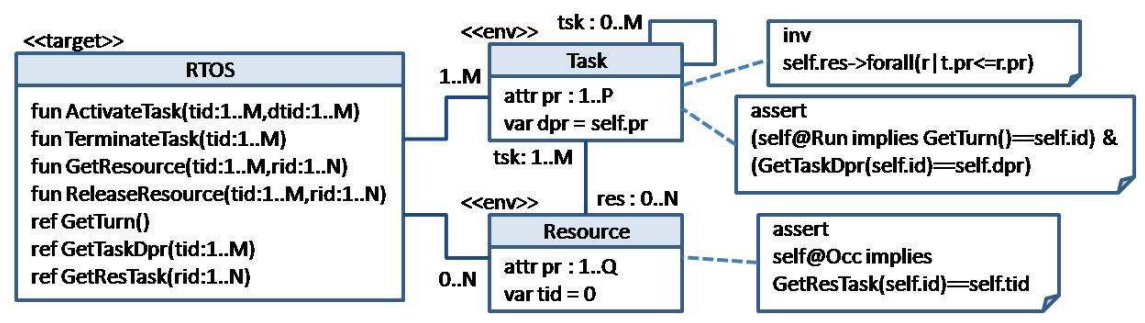

Fig. 3. The class model of an RTOS environment

environment class defines the number of objects which instantiate from the environment class. A variable is a data of an object which can be dynamically changed along with state transitions. It is defined with a default value.

Invariants can be defined for environment classes. They are written in OCL [22]. An invariant defines a constraint on the structure of objects. For example, Task defines an invariant constraining the pattern of links from a task to resources. This invariant reflects the description of the specification that a task can only acquire the resources whose priorities are equal to or higher than that of the task. The OCL expressions in our model is a subset of OCL containing the set operations and the state reference operations.

Assertions are defined for environment classes. An assertion defines a predicate which is checked in each state of objects. In an assertion, the internal values of the target system can be accessed by reference functions to define the consistency between the target system and the environment. For example, Task defines an assertion to check if the variable turn in the RTOS is equal to the identifier of the running task in the environment. It also checks that the runtime priority of a task is the same in the RTOS and the environment. The reference functions GetTurn() and GetTaskDpr() are used to obtain the value of turn and the runtime priority of the task in the RTOS.

\subsection{Statechart models}

In statechart models, we define the state transitions of environment objects expected for the function calls of the target system. Fig. 4 shows the statechart models of Task and Resource. They describe the normal execution sequences of RTOS. A transition occurs by the call of a trigger function. For example, the transition (1) is caused by ActivateTask(). The expression in [] is a guard condition described in OCL. In the model, typical expressions are defined as functions like ExRun ( $)=$ Task $->$ exists $(t \mid t @ R u n)$.

A set of synchronous transitions can be attached to a transition. By synchronous transitions, we can define the transitions of other objects which occur synchronously with the transition of the self object. For example, the transition (2) defines the synchronous transition Run->Rdy : GetRun(). This means: 


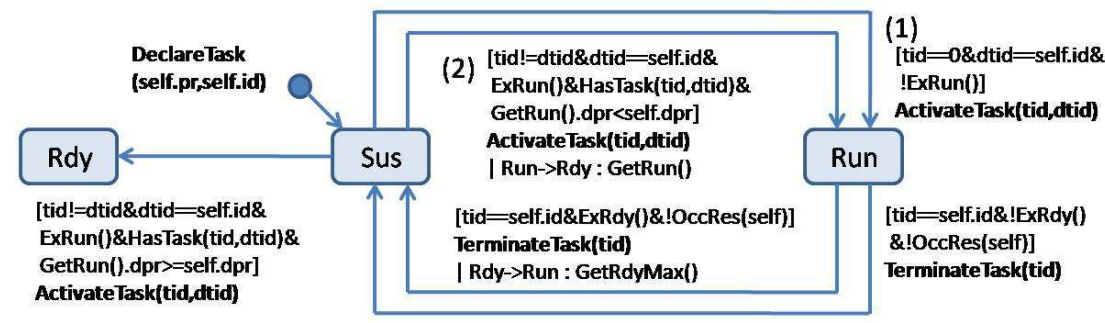

(3) [rid=self.id\&lsRun(tid)\&HasRes(tid,self] ]

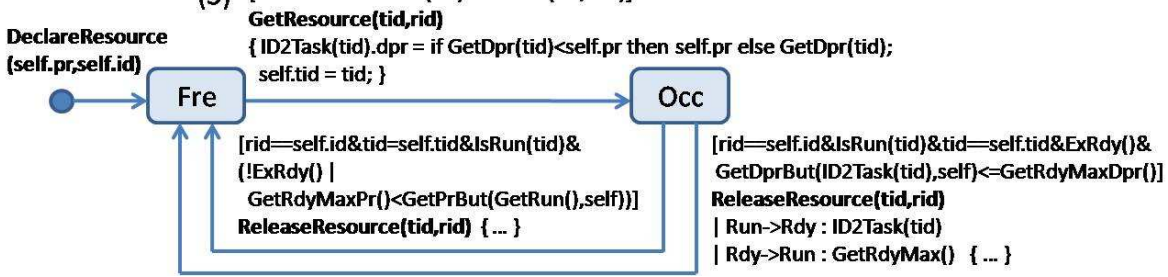

Fig. 4. The statechart model of Task (top) and Resource (bottom)

"Along with the transition of the self object, the Task object obtained by the OCL function GetRun() (the task which is in the state Run) transits from the state Run to Rdy. Currently, asynchronous transitions are not supported.

An action can be attached to a transition. It is a sequence of statements described in \{\} which are executed along with the transition. In an action, variables of objects are updated. For example, the transition (3) defines an action with two statements which update the variables dpr of a task (the runtime priority), and tid of a resource (the task ID occupying the resource), respectively.

\section{Generation of environments}

In this section, we explain the environment generation with the example. As shown in Fig. 5, it is done in three steps: (1) Generation of object graphs, (2) composition of statechart models, and (3) translation into Promela scripts. We also present formal algorithms for (1) and (2) in A.2 and A.3.

\subsection{Generation of object graphs}

Firstly, we generate all the possible object graphs from the class model. An object graph is represented by a set of objects which instantiate from all the classes. Each object holds the values of attributes and associations. The value of an association is a set of objects with which the object links.

We generate object graphs based on a data structure called graph counter which represents an object graph by a vector of natural numbers. By counting 


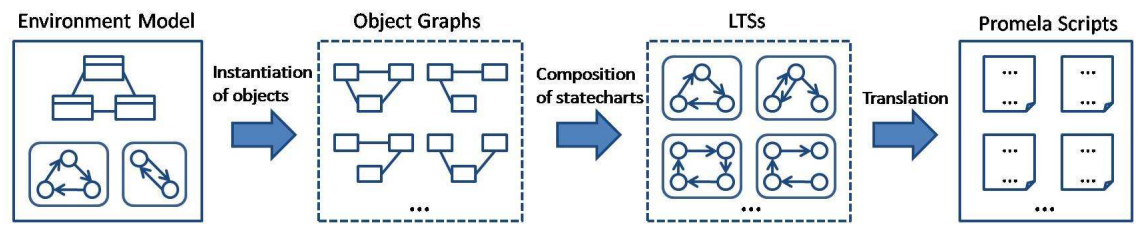

Fig. 5. Environment generation

up the counter, we enumerate all the variation of object graphs. Let us consider the example in Fig. 3 with $\mathrm{M}=2, \mathrm{~N}=1, \mathrm{P}=2$, and $\mathrm{Q}=2$. The graph counter for this model consists of 8 numbers. Each of them corresponds to T1.pr (the task T1's attribute pr), T1.tsk, T1.res, T2.pr, T2.tsk, T2.res, R1.pr and R1.tsk, respectively. For example, the graph counter $\mathrm{L}=\langle 2,3,1,1,4,2,2,3\rangle$ represents an object graph with $\mathrm{T} 1 \cdot \mathrm{pr}=2, \mathrm{~T} 1 . \mathrm{t} \mathrm{sk}=[\mathrm{T} 2], \mathrm{T} 1 . \mathrm{res}=[], \mathrm{T} 2 \cdot \mathrm{pr}=1$, $\mathrm{T} 2 \cdot \mathrm{tsk}=[\mathrm{T} 1, \mathrm{~T} 2], \mathrm{T} 2 \cdot \mathrm{res}=[\mathrm{R} 1], \mathrm{R} 1 \cdot \mathrm{pr}=2$, and R1.tsk $=[\mathrm{T} 1, \mathrm{~T} 2] . \mathrm{L}[1]=2$ means that the T1.pr's value is the 2nd value of the pr's domain $\{1,2\}$. Likewise, $\mathrm{L}[2]=3$ means that the T1.tsk's value is the 3rd value of the tsk's domain $\{[],[\mathrm{T} 1],[\mathrm{T} 2],[\mathrm{T} 1, \mathrm{~T} 2]\}$.

To enumerate all the graphs, we start with the initial counter $\langle 1, \ldots, 1\rangle$ and count it up repeatedly to $\langle 1, \ldots, 1,2\rangle$, and then, $\langle 1, \ldots, 1,3\rangle$. If L [8] reached the maximum number 3 (R1.tsk's domain is $\{[1],[2],[1,2]\}$ ), the next count causes carry over. So, the next counter is $\{1, \ldots, 2,1\}$. We repeat counting until the counter reaches the maximum value $\langle 2,4,2,2,4,2,2,3\rangle$. Along with the counting, we translate the graph counter into an object graph and output it if it satisfies invariants.

The computation time of this algorithm increases exponentially as the multiplicities of associations increase. However, the computation space is limited to $O((A+B) \times C) .(A, B, C$ is the number of attributes, associations, and objects. $)$

\subsection{Composition of statechart models}

Next, we compose the statechart models of all objects in each object graph. The result of the composition is an LTS (Labelled Transition System) [6]. Fig. 6 shows an example of an object graph and its LTS. In the LTS, each state contains the states and variables of all the objects. For example, in the initial state (A), the tasks $\mathrm{T} 1$ and $\mathrm{T} 2$ are in the state Sus and the resource R1 is in the state Fre. The variables dpr of $\mathrm{T} 1$ and $\mathrm{T} 2$ are 1 and 3, respectively. The variable tid of $R 1$ is 0 .

Transitions in statechart models are added to the LTS if their guard conditions are evaluated to true in the LTS state and the object graph. For example, the transition from $(\mathrm{A})$ to $(\mathrm{B})$ by the function ActivateTask $(0,1)(\operatorname{AT}(0,1))$ is added because the guard condition of the transition (1) in Fig. 4 becomes true for the object $\mathrm{T} 1$ and the action argument $(0,1)$ in the state $(\mathrm{A})$. If a transition has synchronous transitions, synchronized objects are obtained by evaluating the 


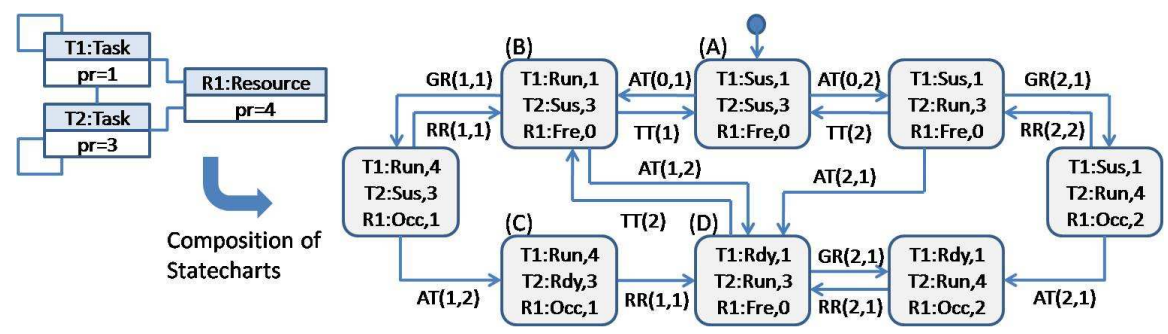

Fig. 6. An object graph and its LTS

OCL expression. The synchronized objects transit along with the transition of the self object. For example, in the transition from (C) to (D) by the function ReleaseResource $(1,1)(\mathrm{RR}(1,1))$, along with the self object R1 transits from Occ to Fre, the synchronized objects T1 and T2 transit from Run and Rdy to Rdy and Run, respectively. This transition also updates the variables of $\mathrm{T} 1$ and R1 by executing the corresponding action.

It could be possible to translate all the statechart models directly into Promela and leave the composition to SPIN. However, we do not do so because the OCL expressions attached with the transitions are hard to express directly in Promela. So, we evaluate the OCL expressions and compose the statechart models at this point.

\subsection{Translation into Promela scripts}

Finally, we translate each LTS into Promera script. A state of LTS contains the states and variables of all objects. In Promela, the states of objects are represented by a label and the variables of objects are represented by the variables of Promela. For example, Fig. 7 shows the Promela script corresponding to the state (D) of the LTS in Fig. 6. In the script, the three states Rdy, Run, and Fre of the three objects T1, T2, and R1 are represented by the label Rdy_Run_Fre. The variables of the three objects are represented by the variables Task1.dpr, Task2.dpr, and Resource1.tid. These variables are checked in the guard conditions of the if-statement and updated appropriately after calling the functions TerminateTask(2) and GetResource $(2,1)$.

The assertion is checked at the beginning of the state. It is a conjunction of the assertions of all the objects. (In this case, only the assertion of two tasks are conjuncted as the assertion of the resource is true.) The three variables ret_GetTurn, ret_GetTaskDpr_1, and ret_GetTaskDpr_2 represent the internal values of the RTOS which are obtained by the reference functions GetTurn(), GetTaskState (1), GetTaskState (2), respectively. These variables are set to the return values of the corresponding functions in the inline function set_ref () before the assertion check. Expressions in the assertions except for these variables are evaluated during the composition of statechart models. 


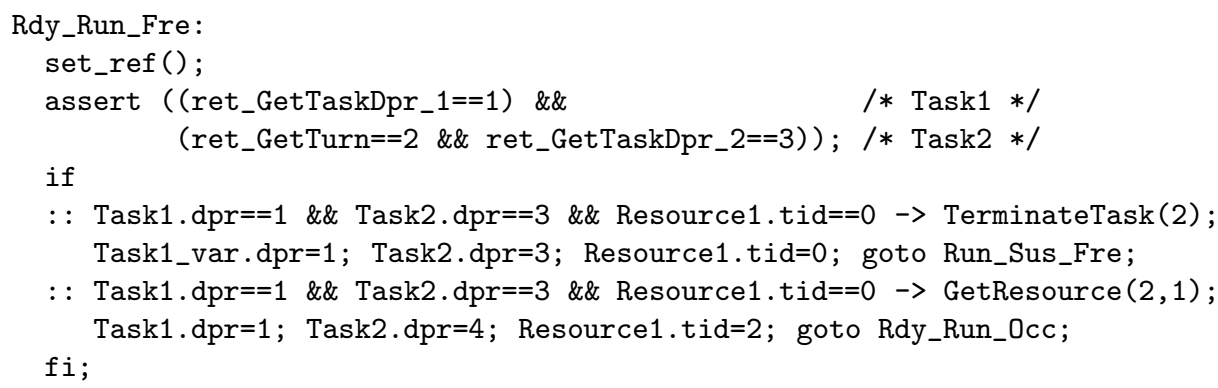

Fig. 7. Promela script corresponding the state (D) in Fig. 6

\subsection{Environment generator}

We implemented the environment generator as a command line tool which inputs an environment model as a text file and outputs all the environments as Promela files. This tool can be applied generally to any systems as long as we observe the interface between the environment model and the target system. (The Promela script of the target system must contain the inline functions corresponding to the trigger functions and reference functions in the environment model.) Fig. 8 shows the architecture of the tool. It mainly consists of three components realizing the three steps of the environment generation: the graph generator, the state composer, and the Promela translator. It also has the invariant filter for checking invariants for object graphs, and the OCL evaluator for evaluating OCL expressions.

\section{$5 \quad$ Experiment}

As an application of our method to a practical system, we conducted an experiment to verify that an RTOS design model conforms to the OSEK/VDX RTOS specification. The design model is implemented in Promela (about 1800 lines) following the approach in [1]. It is implemented as a set of inline functions for the functions ActivateTask, TerminateTask, and so on. For the verification, we constructed the environment model based on the specification. We have presented this model partially in Fig. 3 and Fig. 4. We generated the environments by the environment generator and conducted model checking for some of them in SPIN.

\subsection{Environment generation}

Table. 1 shows the number of generated environments from the model in Fig. 3 with the constants $\mathrm{M}, \mathrm{N}, \mathrm{P}$, and $\mathrm{Q}$ set to 4 , i.e., tasks and resources are created up to 4 and both of them can take priorities up to 4 . We generated environments by moving the number of tasks and resources from 1 to 4 . In the Table. 1, $\mathrm{T}$ is the 


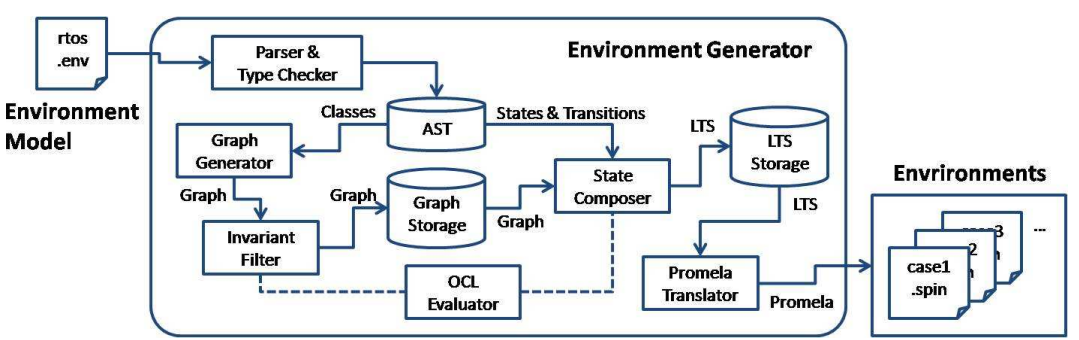

Fig. 8. Architecture of the environment generator

number of tasks and $\mathrm{R}$ is the number of resources. Actually, the specification does not limit the number of tasks and resources and it allows the number of priorities up to 16. In our model, however, we limited the maximum number of objects and priorities to 4 so that the environment generation can be completed within a reasonable time. Even in this limited range, we can capture the important behavior of the RTOS. (See Section 6.)

An important problem when constructing an environment model is to prevent generation of isomorphic environments. An example of a pair of isomorphic environments is: (1) an environment of tasks T1 and T2 with priorities 1 and 2, and (2) an environment of tasks T1 and T2 with priorities 2 and 1. Since the identifiers of tasks do not affect the behavior of the RTOS, this pair of environments brings the same result in model checking. In our method, we can reduce such isomorphic environments by making use of invariants. In the example, the following invariant can be added to the class Task:

Task->forall(t|self.id<t.id implies self.pr<t.pr)

It specifies that the priorities of tasks increases monotonically with the increase of identifiers. By this invariant, we can remove the environment (2). The same kind of invariant can be added to the class Resource. By removing isomorphic environments, we can drastically reduce the number of generated environments. In the cases of two tasks and two resources, we can reduce the number from 850 to 95 ( $88.8 \%$ reduction). In our method, it is the responsibility of users to add appropriate invariants to reduce isomorphic environments.

Currently, the computation time of the generation algorithm grows exponentially with the number of associations. To make it more scalable, we need to compute the set of object graphs, which satisfy invariants, directly from the class model. To realize this, we are currently considering the use of SAT and SMT solvers $[16,4]$.

\subsection{Model checking}

We conducted model checking on some of the generated environments. We selected a representative environment from each case in Table. 1. For example, 


\begin{tabular}{|c||c|c|c|c|}
\hline $\mathrm{R} / \mathrm{T}$ & 1 & 2 & 3 & 4 \\
\hline \hline 0 & $4(0.0 \mathrm{~s})$ & $6(0.0 \mathrm{~s})$ & $4(0.1 \mathrm{~s})$ & $1(0.2 \mathrm{~s})$ \\
\hline 1 & $10(0.1 \mathrm{~s})$ & $40(0.3 \mathrm{~s})$ & $55(1.5 \mathrm{~s})$ & $26(3.5 \mathrm{~s})$ \\
\hline 2 & $10(0.2 \mathrm{~s})$ & $95(1.5 \mathrm{~s})$ & $245(15.7 \mathrm{~s})$ & $196(99.5 \mathrm{~s})$ \\
\hline 3 & $5(0.2 \mathrm{~s})$ & $100(6.8 \mathrm{~s})$ & $425(169.2 \mathrm{~s})$ & N/A \\
\hline 4 & $1(1.0 \mathrm{~s})$ & $39(66.3 \mathrm{~s})$ & N/A & N/A \\
\hline
\end{tabular}

Table 1. The number of generated environments (CPU:2.4GHz, Memory:4.0GB)

for the 95 cases in $\mathrm{T}=2$ and $\mathrm{R}=2$, we selected the case No. $95 / 2=47$. Table. 2 shows the results of model checking. As well as the check results (" $\bigcirc$ " for no errors and " $\times$ " for at least one error), it shows the structures of object graphs, consumed time and memories. (Times include the compilation time and the verification time.) As the memory consumption indicates, we were able to check all the cases without causing state explosion. This is thanks to the structural decomposition of the environment. If we naively conduct model checking with a non-deterministic environment, we cannot even check the environment of up to 2 tasks and 2 resources causing state explosion with 4.0 Gbyte of memory.

Among 17 cases, 6 cases were unsuccessful. All the failures were caused by assertion violations. Specifically, the runtime priority of a task was inconsistent between the environment and the RTOS. In our method, we can make use of the table to conduct difference analysis to find the source of a bug. By overlooking the table, we can see that the check fails only if there are at least two tasks. With a closer look, we can notice that the check fails only if there is at least one task which is linked with more than one resources. Based on this information, we can presume that the resource handling of the RTOS is incorrect, especially when a task tries to acquire more than one resources.

By examining the counter example traces output by SPIN, we found that the bug was contained in the function GetResource ( ). It is a function called from a task when the task acquires a resource. Correctly, the function must raise the runtime priority of the task to the resource priority only when the runtime priority is lower than the resource priority. (This mechanism is called ceiling priority protocol.) But the function changed the runtime priority every time the task acquires a resource regardless of the resource priority. This bug caused the inconsistency of the runtime priority between the environment and the RTOS. (For example, if $\mathrm{T} 1$ first acquires R1 of priority 4 and then acquires $\mathrm{R} 2$ of priority 3 , the environment expect the runtime priority of $\mathrm{T} 1$ to be 4 . In the RTOS, however, it was incorrectly changed to 3.) This inconsistency occurs only when a task tries to acquire more than one resources. This result coincides with our presumption based on the difference analysis.

When model checking in SPIN, it is not always easy to pinpoint the source of the bug only from the information of the counter example traces. Our method, however, can provide additional information as a form of a table by which we can conduct difference analysis based on graph structures. This is one of the 


\begin{tabular}{|c|c|c|c|c|c|c|c|c|c|c|c|c|c|c|c|c|}
\hline \multirow{2}{*}{\begin{tabular}{|l|}
$\mathrm{T}, \mathrm{R}$ \\
\end{tabular}} & \multirow{2}{*}{\begin{tabular}{|l|} 
No. \\
\end{tabular}} & \multirow{2}{*}{\multicolumn{4}{|c|}{\begin{tabular}{|c|c|c|c|}
\multicolumn{3}{|c|}{ Task.pr } \\
T1 & T2 & T3 & T4 \\
\end{tabular}}} & \multicolumn{4}{|c|}{ Task.res } & \multicolumn{4}{|c|}{ Resource.pr } & \multirow[t]{2}{*}{ Result } & \multirow{2}{*}{$\begin{array}{l}\text { Time } \\
(\mathrm{sec})\end{array}$} & \multirow{2}{*}{$\begin{array}{l}\text { Memory } \\
\text { (Mbyte) }\end{array}$} \\
\hline & & & & & & $\mathrm{T} 1$ & $\mathrm{~T} 2$ & T3 & $3 \mathrm{~T} 4$ & & $\overline{\mathrm{R} 2[1}$ & R3 & $\mathrm{R} 4$ & & & \\
\hline 1,0 & 2 & $2 \mid$ & & & & [] & & & & & & & & $\mathrm{O}$ & 3.3 & 2.70 \\
\hline 1,1 & 5 & 2 & & & & [1] & & & & \begin{tabular}{l|l}
3 \\
\end{tabular} & & & & 0 & 3.4 & 2.70 \\
\hline 1,2 & 5 & 1 & & & & {$[1,2]$} & & & & 1 & 4 & & & $x$ & 3.6 & 2.79 \\
\hline 1,3 & 2 & 1 & & & & {$[1,2,3]$} & & & & 1 & 2 & 4 & & $x$ & 4.0 & 2.89 \\
\hline 1,4 & 1 & 1 & & & & {$[1,2,3,4]$} & & & & 1 & 2 & 3 & 4 & $x$ & 5.2 & 3.09 \\
\hline 2,0 & 3 & 2 & 3 & & & U] & & & & & & & & $\mathrm{O}$ & 3.6 & 2.89 \\
\hline 2,1 & 20 & 3 & 4 & & & [1] & & & & 4 & & & & O & 3.7 & 2.89 \\
\hline 2,2 & 47 & 2 & 3 & & & [1] & {$[2]$} & & & 2 & 4 & & & $\mathrm{O}$ & 4.0 & 3.09 \\
\hline 2,3 & 50 & 1 & 3 & & & {$[1,2,3]$} & [3] & & & 1 & 3 & 4 & & $x$ & 5.8 & 3.38 \\
\hline 2,4 & 19 & 1 & 2 & & & {$[1,2,3,4]$} & {$[2,3]$} & & & 1 & 2 & 3 & 4 & $x$ & 10.4 & 3.77 \\
\hline 3,0 & 2 & 1 & 2 & 4 & & {[]} & {[]} & & & . & & & & 0 & 4.2 & 3.09 \\
\hline 3,1 & 27 & 1 & 2 & 4 & & [1] & [1] & & & $2 \mid$ & & & & O & 4.9 & 3.58 \\
\hline 3,2 & 122 & 1 & 2 & 3 & & [1] & [] & {$[2]$} & & $1 \mid$ & \begin{tabular}{l|l}
3 \\
\end{tabular} & & & 0 & 5.3 & 3.87 \\
\hline 3,3 & 212 & 1 & 2 & 3 & & [2] & [1] & [3] & & 2 & 3 & 4 & & 0 & 6.3 & 4.36 \\
\hline 4,0 & 1 & 1 & 2 & 3 & 4 & {[]} & 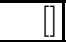 & $\overline{[5]}$ & & & & & & 0 & 5.6 & 4.06 \\
\hline 4,1 & 13 & 1 & 2 & 3 & 4 & [1] & [] & [1] & & 3 & & & & 0 & 7.8 & 5.53 \\
\hline 4,2 & 98 & \begin{tabular}{l|l}
1 \\
\end{tabular} & $2 \mid$ & 3 & 4 & [1] & {$[1]$} & {$[1,2]$} & & \begin{tabular}{l|l}
3 \\
\end{tabular} & 4 & & & $x$ & 11.1 & 4.26 \\
\hline
\end{tabular}

Table 2. Results of model checking

advantages of our method to structurally decompose the whole environment into individuals.

\section{Discussion}

Our method, where a limited range of environment is constructed, stands in contrast to the usual method where a non-deterministic environment is filtered by LTL formula. As we stated in introduction, there is a trade-off between the two methods, i.e., the usual method has an advantage in the coverage of verification, while our method has an advantage in the simplicity and precision of property description and avoidance of state explosion problem. Our method becomes more advantageous for the verification of the systems whose environment has a lot of structural variations. RTOS is a good example of such systems. If the environment has wider structural variations, it can be separated more finely into individuals. As a result, we are more likely to avoid state explosion and more benefit from the structural difference analysis.

To improve the coverage, we need to extend the variation of the environment model as much as possible depending on the computation time of environment generation and model checking. But as it is hard to cover all the cases, we need some criteria as to how far we should extend the variation of the environment model. In our experience, crucial errors concerning the behavioral logic of the system can be discovered even with a small number of objects, and the errors newly discovered by increasing the number of objects are only those concerning 
system boundaries (such as generating objects which exceeds the limit of an array). So, one of the criteria should be to clarify the important properties of the system and cover at least the variations which can observe the satisfaction of the properties. For example, the important properties about the behavioral logic of RTOS are: (1) Without resources, the task of higher priority must be executed before that of lower priority, (2) If a task occupies multiple resources, its runtime priority is set to the maximum priority of the resources, (3) With resources, the task of higher runtime priority must be executed before that of lower runtime priority. To check these properties, we need at least 2 tasks and 2 resources, i.e., (1) requires 2 tasks, (2) requires 1 tasks and 2 resources, and (3) requires 2 tasks and 2 resources. In this way, we need to clarify the necessary minimum of the variations depending on the properties to check and define the parameter ranges sufficiently wide to cover the variations.

For behavioral coverage, we further need to conduct verification of abnormal execution and interrupt handling. To verify interrupt handling, we need to extend the environment model so that it can deal with multiple processes since interleavings occur between the executions of tasks and the interrupt handler.

Another advantage of our method is that it has a potential for parallelizing model checking. Since all the environments are structurally different from each other, they can be checked independently of others. So, it is effective to check all the cases parallelly by distributing them on a PC cluster. To realize this, we need to address two problems. The first one is load-balancing, i.e., how to distribute all the environments equally to each PC. For this problem, we consider it effective to distribute them based on the length of Promela files. This is because most of the time for checking an environment is occupied by the compilation of the Promela file of the environment. The second one is data-mining, i.e., how to retrieve useful information from the large amounts of check results. For this, we consider it effective to make use of a relational database. By storing the results in the database, we can retrieve necessary information by issuing query on environment structures.

\section{Related work}

Many work propose methods to verify UML models in SPIN by translating statechart models into Promela [19,9,14].Our method also applies model checking to statechart models, but our motivation is totally different from these works in that we are using statechart models for describing environments, not the target system itself.

O. Tkachuk, et al. [20] proposes Bandera Environment Generator (BEG) which automatically generates the environment for the verification of Java programs in Bandera. It has been applied to commercial software [21] and has also used as the core tool for the environment generator for web application domain [17]. In BEG, the environment is generated from the specifications of the environment written by the user, called environment assumptions, or by analyzing the programs which implements the environment. The environment assump- 
tions are described as the sequences of the method calls in the form of regular expressions. This approach corresponds to describing a single instance of the environment model in our method. In our method, we can describe a set of the instances as a class model and automatically generate all the possible instances based on the variations in the model.

P. Parizek, et al. [12] proposes a method to verify Java components by Java PathFinder (JPF) and a protocol checker. The protocols of the components are defined by ADL (Architecture Description Language) from which the environment for the components is constructed. This method allows to describe the environment structure by ADL. Compared to this work, our method further describe the variation of the structure using the parameters in the class model.

J. Penix, et al. $[15,13]$ verifies the time partitioning of DEOS RTOS by SPIN. M. Dwyer et al. [5] verifies partial systems described in Ada by translating them into SPIN. In these works, environments are obtained by filtering a universal environment with assumptions described in LTL. This approach is effective when the assumptions can be described simply, but shows weakness when describing precise behavior of environments due to the accumulation of complex LTL assumptions. Compared to these works, we describe the specific behavior of an environment from the beginning using statechart models instead of incrementally refining the universal environment by assumptions. The use of statechart models facilitates the description of environments because the abstraction level is lifted to the familiar level for users.

C. Boyapati et al. [3] developed the Korat tool to automatically generate test cases for unit-testing Java methods. To test a method, it automatically generates all the structural variations of the input object based on the pre-condition. Then the method is tested against the post-condition for all the generated inputs. Our work is close to this work in that the test environment (input) is automatically generated. But our work is more suited for the integration or system-wide testing where the correctness of a system is checked against a sequence of function calls described in statechart models.

Concerning parallel model checking, there are many techniques based on multi-cores [7, 8] and clusters [2]. Compared to these techniques where the search algorithm is parallelized, our method can be parallelized based on separation of data, i.e., we can divide the whole environment into individuals by changing its data settings.

\section{Conclusion}

In this paper, we presented a method for modeling and generating environments for model checking embedded systems. We presented a tool to automatically generate Promela/SPIN scripts from the environment model. As an application to a practical system, we conducted a verification of an OSEK/VDX RTOS design model. In the experiment, we were able to generate sufficient variations of environments efficiently for checking important properties of RTOS. We also confirmed the effectiveness of difference analysis based on the environment struc- 
tures. Future work is to develop a distributed parallel model checking framework based on our method.

\section{References}

1. Toshiaki Aoki. Model Checking Multi-Task Software on Real-Time Operating Systems. In ISORC, pages 551-555. IEEE Computer Society, 2008.

2. Jiří Barnat, Luboš Brim, and Petr Ročkai. DiVinE 2.0: High-Performance Model Checking. In 2009 International Workshop on High Performance Computational Systems Biology (HiBi 2009), pages 31-32. IEEE Computer Society Press, 2009.

3. Chandrasekhar Boyapati, Sarfraz Khurshid, and Darko Marinov. Korat: automated testing based on Java predicates. In ISSTA, pages 123-133, 2002.

4. Leonardo Mendonça de Moura, Bruno Dutertre, and Natarajan Shankar. A Tutorial on Satisfiability Modulo Theories. In Werner Damm and Holger Hermanns, editors, CAV, volume 4590 of Lecture Notes in Computer Science, pages 20-36. Springer, 2007.

5. Matthew B. Dwyer and Corina S. Pasareanu. Filter-Based Model Checking of Partial Systems. In SIGSOFT FSE, pages 189-202, 1998.

6. J. Magee et al. Concurrency: State models \& Java programs. Wiley, 1999.

7. Gerard J. Holzmann and Dragan Bosnacki. The Design of a Multicore Extension of the SPIN Model Checker. IEEE Trans. Software Eng., 33(10):659-674, 2007.

8. Gerard J. Holzmann, Rajeev Joshi, and Alex Groce. Swarm Verification. In ASE, pages 1-6. IEEE, 2008.

9. Johan Lilius and Ivan Paltor. vUML: A tool for verifying UML models. In $A S E$, pages 255-258, 1999.

10. OMG. Unified Modeling Language. http://www.uml.org/, 1989.

11. OSEK/VDX. OSEK/VDX Operating System Specification 2.2.3. http://portal.osek-vdx.org/, 2005.

12. Pavel Parizek and Frantisek Plasil. Partial Verification of Software Components: Heuristics for Environment Construction. In EUROMICRO-SEAA, pages 75-82. IEEE Computer Society, 2007.

13. Corina S. Pasareanu. DEOS Kernel: Environment Modeling using LTL Assumptions. Nasa ames technical report nasa-arc-ic-2000-196, NASA Ames Research Center, 2000.

14. Patrizio Pelliccione, Paola Inverardi, and Henry Muccini. CHARMY: A Framework for Designing and Verifying Architectural Specifications. IEEE Trans. Software Eng., 35(3):325-346, 2009 .

15. John Penix, Willem Visser, Seungjoon Park, Corina S. Pasareanu, Eric Engstrom, Aaron Larson, and Nicholas Weininger. Verifying Time Partitioning in the DEOS Scheduling Kernel. Formal Methods in System Design, 26(2):103-135, 2005.

16. Mukul R. Prasad, Armin Biere, and Aarti Gupta. A survey of recent advances in SAT-based formal verification. STTT, 7(2):156-173, 2005.

17. Sreeranga P. Rajan, Oksana Tkachuk, Mukul R. Prasad, Indradeep Ghosh, Nitin Goel, and Tadahiro Uehara. WEAVE: WEb Applications Validation Environment. In ICSE Companion, pages 101-111. IEEE, 2009.

18. Ranjit Jhala and Rupak Majumdar. Software model checking. ACM Comput. Surv., 41(4), 2009.

19. Timm Schäfer, Alexander Knapp, and Stephan Merz. Model checking UML state machines and collaborations. Electr. Notes Theor. Comput. Sci., 55(3), 2001. 
20. Oksana Tkachuk, Matthew B. Dwyer, and Corina S. Pasareanu. Automated Environment Generation for Software Model Checking. In ASE, pages 116-129. IEEE Computer Society, 2003.

21. Oksana Tkachuk and Sreeranga P. Rajan. Application of automated environment generation to commercial software. In Lori L. Pollock and Mauro Pezzè, editors, ISSTA, pages 203-214. ACM, 2006.

22. J. Warmer and A. Kleppe. The Object Constraint Language: Precise Modeling with UML. Addison-Wesley, 1999.

\section{A Formal definitions and algorithms}

\section{A.1 Environment model}

Definition 1 (Environment model). An environment model EM is defined as:

$$
E M=(I, \mathcal{C}, \mathcal{S})
$$

$I$ is a target system. $\mathcal{C}=\left\{C_{1}, C_{2}, \ldots\right\}$ is the set of classes. $\mathcal{S}=\left\{S C_{1}, S C_{2}, \ldots\right\}$ is the set of statechart models.

Definition 2 (Target system). The target system I is defined as:

$$
I=(\text { Fun, Ref, Arg })
$$

The sets Fun and Ref are the set of trigger functions and reference functions, respectively. The mapping Arg: $F \times 2^{\text {Val }}$ relates a function to the domain (variation) of its argument. (For simplicity, we present the definition where a function has only one argument.) The set Val is the set of values.

Definition 3 (Classes). The class $C_{i} \in \mathcal{C}$ is defined as:

$$
C_{i}=\left(X_{i}, Y_{i}, V_{i}, \text { Size }_{i}, \text { Dom }_{i}, \text { Def }_{i}, \text { Inv }_{i}, \text { Assr }_{i}\right)
$$

The sets $X_{i}$ and $Y_{i}$ are the sets of attributes and associations, respectively. We define the union $X_{i} \cup Y_{i}$ as $U_{i}=\left\{u_{i 1}, u_{i 2}, \ldots\right\}$, and call them members of the class $C_{i}$. The set $V_{i}=\left\{v_{i 1}, v_{i 2}, \ldots\right\}$ is the set of variables. Size $e_{i} \in N$ is the number of objects which instantiate from the class. The mapping Dom Do $_{i} U_{i} \rightarrow 2^{\text {Val }}$ relates a member to the set of values which represents the domain (variation) of the member. We represent each element in $\operatorname{Dom}_{i}\left(u_{i j}\right)$ as $d_{i j k}(k=1, \ldots)$. The mapping Def $f_{i}: V_{i} \rightarrow$ Val relates a variable to its default value. We represent each element in Defi $f_{i j}\left(v_{i j}\right)$ as $e_{i j}$. The expression $\operatorname{Inv}_{i}, A s s r_{i} \in E x p$ are an invariant and an assertion. Exp is the set of expressions (Val $\subset$ Exp).

In this definition, we omit the definition of multiplicities of associations. The variation of links for an association is directly defined by $D o m_{i}$.

Definition 4 (Statechart models). Let $S$ be the set of states. For the class $C_{i}$, the statechart model $S C_{i}$ is defined as:

$$
S C_{i}=\left(S_{i}, s_{0 i}, A_{i}, T_{i}\right)
$$


The set $S_{i} \subset S$ is the set of states. The state $s_{0 i} \in S_{i}$ is the initial state. The set $A_{i}$ is a set of actions. The set $T_{i}$ is the set of transitions. For a transition $\left(s_{1}, s_{2}, g, f, a, s t\right) \in T_{i}, s_{1}, s_{2} \in S_{i}$ are the source and destination states, respectively. $g \in$ Exp is a guard condition. $f \in$ Fun is a trigger function. $a \in A_{i}$ is an action. st is the set of synchronous transitions. For a synchronous transition $\left(t_{1}, t_{2}, x\right) \in$ st, $t_{1}, t_{2} \in S$ are the source and destination states, respectively. $x \in \operatorname{Exp}$ is the expression representing the synchronized objects.

\section{A.2 Generation of object graphs}

Definition 5 (Object graphs). Let $O_{i}=\left\{o_{i 1}, o_{i 2}, \ldots\right\}$ be the set of objects which instantiate from the class $c_{i}\left(\left|O_{i}\right|=S i z e\left(c_{i}\right)\right)$. Let $\mathcal{G}$ be the set of object graphs. An object graph $G \in \mathcal{G}$ is defined as:

$$
G=\left\{g_{i j k}|i=1 . .| C|, j=1 . .| O_{i}|, k=1 . .| U_{i j} \mid\right\}
$$

The value $g_{i j k}$ represents the value of the member $u_{i k}$ of the object $o_{i j}$.

The set of object graphs $\mathcal{G}$ is computed as follows. Firstly, we define the graph counter $Z=\left\langle z_{1}, z_{2}, \ldots\right\rangle$ as a vector of length $M=\sum_{i=1}^{|C|}\left(\left|O_{i}\right| \times\left|U_{i}\right|\right)$. The correspondence between object members and graph counter elements are defined by the function $\operatorname{Pos}(i, j, k)=\left(\sum_{n=1}^{i-1}\left|O_{n}\right| \times\left|U_{n}\right|\right)+\left|U_{i}\right| \times(j-1)+k$. If $\operatorname{Pos}(i, j, k)=p, z_{p}$ corresponds to the member $u_{i j k}$. For each $z_{i}$, we define its maximum value as $\operatorname{Max}_{i}$. If $\operatorname{Pos}(i, j, k)=p, \operatorname{Max}_{p}$ is equal to $\left|\operatorname{Dom}\left(u_{i k}\right)\right|$.

Then, we define a function GetGraph which generates an object graph from the graph counter.

$\operatorname{Get} \operatorname{Graph}(Z)=\left(G, Z^{\prime}\right)$

where

$$
\begin{aligned}
& G_{i j k}=d_{i j m}\left(m=L[\operatorname{Pos}(i, j, k)], i=1 . .|C|, j=1 . .\left|O_{i}\right|, k=1 . .\left|U_{i}\right|\right) \\
& Z^{\prime}= \begin{cases}\left\langle z_{1}, \ldots, z_{M}+1\right\rangle & \text { if } z_{M}<\operatorname{Max}_{M} \\
\left\langle z_{1}, \ldots, z_{i}+1,1, \ldots, 1\right\rangle & \text { if } z_{i}<\operatorname{Max}_{i}, z_{j}=\operatorname{Max}_{j}(j=i+1 . . M) \\
\langle 1, \ldots, 1\rangle & \text { if } z_{i}=\operatorname{Max}_{i}(i=1 . . M)\end{cases}
\end{aligned}
$$

$G$ and $Z^{\prime}$ are the generated object graph and the next graph counter, respectively. The last case of $Z^{\prime}$ means that the counter returns to $\langle 1, \ldots, 1\rangle$ when it reaches maximum. By this, we know the end of counting.

Finally, the set $\mathcal{G}$ is obtained the following algorithm. In the algorithm, the mapping $\operatorname{Eval}_{G}[o]: \operatorname{Exp} \rightarrow$ Val relates an expression to a value which is obtained by evaluating the expression in the context of a graph $G$ and an object $o$.

1. Let $\mathcal{G}=\{\}$ and $Z=\langle 1, \ldots, 1\rangle$.

2. Let $(G, Z)=\operatorname{Get} \operatorname{Graph}(Z)$

3. If $G$ satisfies invariants, i.e., $\operatorname{Eval}_{G}\left[o_{i j}\right]\left(\operatorname{Inv}_{i}\right)$ is a value representing true for all $i$ and $j$, let $\mathcal{G}=\{G\} \cup \mathcal{G}$.

4. If $Z \neq\langle 1, \ldots, 1\rangle$, goto 2 . 


\section{A.3 Composition of statecharts}

Definition 6 (Labelled transition systems). An LTS L is defined as:

$$
L=\left(P, p_{0}, Q, R, B\right)
$$

The set $P=\left\{p_{0}, p_{1} \ldots\right\}$ is a set of composite states. In a composite state $p$, the state of the object $o_{i j}$ and the value of the variable $v_{i j k}$ are defined as $p[i, j, 0]$ and $p[i, j, k]$, respectively. (Note that $k=1 \ldots$ ) The state $p_{0}$ is the initial state. The set $Q$ is the set of labels. For a label $(f, w) \in Q, f$ and $w$ are a trigger function and its argument, respectively. The set $R$ is the set of transitions. For a transition $r=\left(t_{1}, t_{2}, q\right) \in R, t_{1}, t_{2}$, and $q$ are a source state, a destination states, and a label, respectively. The set $B=\left\{b_{0}, b_{1}, \ldots\right\}$ is the set of assertions. Each $b_{i}$ is the assertion defined for the state $p_{i}$.

For an object graph $G$, an LTS $L$ is computed by the following algorithm. In the algorithm, the mapping $\operatorname{Eval}_{G}[o][p][w]: \operatorname{Exp} \rightarrow$ Val relates an expression to a value which is obtained by evaluating the expression in the context of a graph $G$, a state $p$, an object $o$, and an argument $w$. The mapping $A E v a l_{G}[o][p]$ : $E x p \rightarrow E x p$ relates an assertion to an expression which is obtained by evaluating the assertion in the context of a graph $G$, a state $p$, and an object $o$. (The result expression may contain reference functions.) The mapping $\operatorname{Exec}_{G}[o][p][w]$ : $A \times V \rightarrow V a l$ relates an action and a variable to the value of the variable which is obtained by evaluating the action in the context of a graph $G$, a state $p$, and an $\operatorname{argument} w$. For simplicity, we present the algorithm for the case where the expression of a synchronous transition always evaluates to a single object.

1. Let $P=Q=B=\{\}$.

2. Define the initial state $p_{0}$ such that $p_{0}[i, j, 0]=s_{0 i}$ and $p_{0}[i, j, k]=D e f_{i}\left(y_{i k}\right)$.

3. Let $p=p_{0}$ and $P_{i}=\left\{p_{0}\right\}$ ( $p$ is a temporal variable).

4. For each object $o_{i j}$, for each transition $\left(p_{i j 0}, s, g, f, a, s t\right) \in T_{i}$, and for each argument $w \in \operatorname{Arg}(f)$, do the following steps.

(a) If the guard condition $g$ is true, i.e., the expression $\operatorname{Eval}_{G}\left[o_{i j}\right][p][w](g)$ is a value representing true, create a new state $q$ with $q[m, n, 0]$ defined as follows:

- If $m=i$ and $n=j$, then $s$.

- If there exists a synchronous transition $\left(x, t_{1}, t_{2}\right) \in s t$ such that the target object $x$ is $o_{m n}$, i.e., the expression $\operatorname{Eval}_{G}\left[o_{i j}\right][p][w](x)$ is a value representing $o_{m n}$ and $p[m, n, 0]=t_{1}$, then $t_{2}$.

- Otherwise, $p[m, n, 0]$.

For all cases, $q[m, n, l]$ is defined as the value $\operatorname{Exec}_{G}\left(o_{m n}, a, v_{m l}\right)$.

(b) Let $Q=\{(f, w)\} \cup Q$ and $R=\{(p, q,(f, w))\} \cup R$.

(c) If $q \notin P$, let $P=\{q\} \cup P$ and $p=q$, and go to 4 .

5. For each $p_{i} \in P$, let $b_{i}=\bigwedge_{j, k} A E v a l_{G}\left[o_{j k}\right]\left[p_{i}\right]\left(A s s r_{j}\right)$ and $B=\left\{b_{i}\right\} \cup B$. 